\title{
Practical Cryptanalysis of ARMADILLO2
}

\author{
María Naya-Plasencia ${ }^{1, \star}$ and Thomas Peyrin ${ }^{2, \star \star}$ \\ 1 University of Versailles, France \\ maria.naya-plasencia@prism.uvsq. fr \\ 2 Division of Mathematical Sciences, School of Physical and Mathematical Sciences, \\ Nanyang Technological University, Singapore \\ thomas.peyrin@gmail.com
}

\begin{abstract}
The ARMADILLO2 primitive is a very innovative hardwareoriented multi-purpose design published at CHES 2010 and based on data-dependent bit transpositions. In this paper, we first show a very unpleasant property of the internal permutation that allows for example to obtain a cheap distinguisher on ARMADILLO2 when instantiated as a stream-cipher. Then, we exploit the very weak diffusion properties of the internal permutation when the attacker can control the Hamming weight of the input values, leading to a practical free-start collision attack on the ARMADILL02 compression function. Moreover, we describe a new attack so-called local-linearization that seems to be very efficient on datadependent bit transpositions designs and we obtain a practical semifree-start collision attack on the ARMADILL02 hash function. Finally, we provide a related-key recovery attack when ARMADILLO2 is instantiated as a stream cipher. All collision attacks have been verified experimentally, they require negligible memory and a very small number of computations (less than one second on an average computer), even for the high security versions of the scheme.
\end{abstract}

Keywords: ARMADILL02, hash function, stream-cipher, MAC, cryptanalysis, collision.

\section{Introduction}

Hash functions are among the most important and widely spread primitives in cryptography. Informally a hash function $H$ is a function that takes an arbitrarily long message as input and outputs a fixed-length hash value of size $n$ bits. The classical security requirements for such a function are collision resistance and (second)-preimage resistance. Namely, it should be impossible for an adversary to find a collision (two different messages that lead to the same hash value) in less than $2^{n / 2}$ hash computations, or a (second)-preimage (a message hashing to a given challenge) in less than $2^{n}$ hash computations. In general, a hash function $H$

\footnotetext{
* Supported by the French Agence Nationale de la Recherche through the SAPHIR2 project under Contract ANR-08-VERS-014.

** Supported by the Lee Kuan Yew Postdoctoral Fellowship 2011 and the Singapore National Research Foundation Fellowship 2012.
}

A. Canteaut (Ed.): FSE 2012, LNCS 7549, pp. 146-162, 2012.

(C) International Association for Cryptologic Research 2012 
is built from an iterative use of a $n$-bit output compression function $h$ in a MerkleDamgård-like operating mode 644. The compression function takes a chaining variable $C V$ (fixed to an initial value $I V$ at the beginning) and a message block $M$ as inputs and in order to allow security proofs on the operating mode, one requires the same security properties as a hash function, namely collision and (second)-preimage resistance. However, the compression function allows several flavors of security properties depending on how well the attacker can control the chaining variable:

- free-start collision: the attacker fully controls the chaining variable, i.e. both its value and difference

- semi-free-start collision: the attacker control partially the chaining variable, i.e. only its value, and the difference is null

- collision: the attacker does not control the chaining variable, the value is defined by the $I V$ and the difference is null

For all three flavors, it should be impossible for an adversary to find a collision in less than $2^{n / 2}$ compression function computations. Note that free-start collision is required as necessary assumption regarding the compression function in the Merkle-Damgård-like security proofs. Moreover, a semi-free-start collision means there exists initial values $I V$ for which it is possible to find collisions for the hash function. Therefore, both these two notions are very important and should be verified for a secure compression function.

ARMADILLO2 2] is a very novel primitive dedicated to hardware, defining a FIL-MAC, a stream cipher and a hash function. Originally, two versions were proposed, ARMADILLO and ARMADILLO2, the later being the recommended one. A key recovery attack on ARMADILLO was rapidly published by a subset of the designers [9. ARMADILL02 remained unbroken until Abdelraheem et al. [1] found a meet-in-the-middle technique that allows to invert the ARMADILL02 main function. This cryptanalysis eventually led to a key recovery attack on the FIL-MAC and the stream cipher, and a (second)-preimage attack on the hash function. However, while being the first weakness published on ARMADILL02, this work is an improved meet-in-the-middle technique, therefore requiring a lot of computations and memory, often close to the generic complexity. For example, the preimage attack on the 256-bit output hash function requires either $2^{208}$ computations and $2^{205}$ memory or $2^{249}$ computations and $2^{45}$ memory. With its data-dependent bit transpositions and original compression function construction, ARMADILL02 is clearly not following the classical design trends for symmetric-key primitives (for example RC5 [7] and RC6 [8] use data-dependent rotations, while IDEA [5] use data-dependent multiplication). As a consequence, it would be interesting to look at this proposal without necessarily relying on known cryptanalysis techniques.

Our Contributions. In this paper, we first observe the very unpleasant property that the parity bit is preserved through all ARMADILLO2 internal permutations. This allows us for example to derive a very cheap distinguisher for the stream-cipher. Then, we analyze the differential diffusion of the permutations and we provide practical free-start collision attacks for all versions of the 
compression function of ARMADILL02. We extend our results by introducing a new technique, the local linearization, that seems very efficient against datadependent bit transpositions. This method led us to practical semi-free-start collision attacks for all versions of ARMADILL02. All attacks require very few computations (at most $2^{10.2}$ operations for 256-bit output version) and negligible memory. Moreover, our implementations validate our techniques and we provide collision examples. Finally, we provide a related-key recovery attack when ARMADILLO2 is instantiated as a stream cipher.

\section{The ARMADILLO2 Function}

We let $X[i]$ denote the $i$-th bit of a word $X$. Let $C$ be an initial vector of size $c$ and $U$ be a message block of size $m$. The size of the register $(C \| U)$ is $k=c+m$, where $\|$ denotes the concatenation operation. The internal ARMADILL02 function transforms the vector $(C, U)$ into $\left(V_{c}, V_{t}\right)$ as described in Figure 1. $\left(V_{c}, V_{t}\right)=$ ARMADILLO2 $(C, U)$. The internal ARMADILL02 function relies on a parameterized permutation on $k$ bits $Q$, instantiated by $Q_{U}$ and $Q_{X}$, where $U$ is a $m$-bit parameter and $X$ is a $k$-bit parameter.

Let $\sigma_{0}$ and $\sigma_{1}$ be two fixed bitwise permutations of size $k$. In [2], the permutations are not specifically defined but some criteria they should fulfill is given.

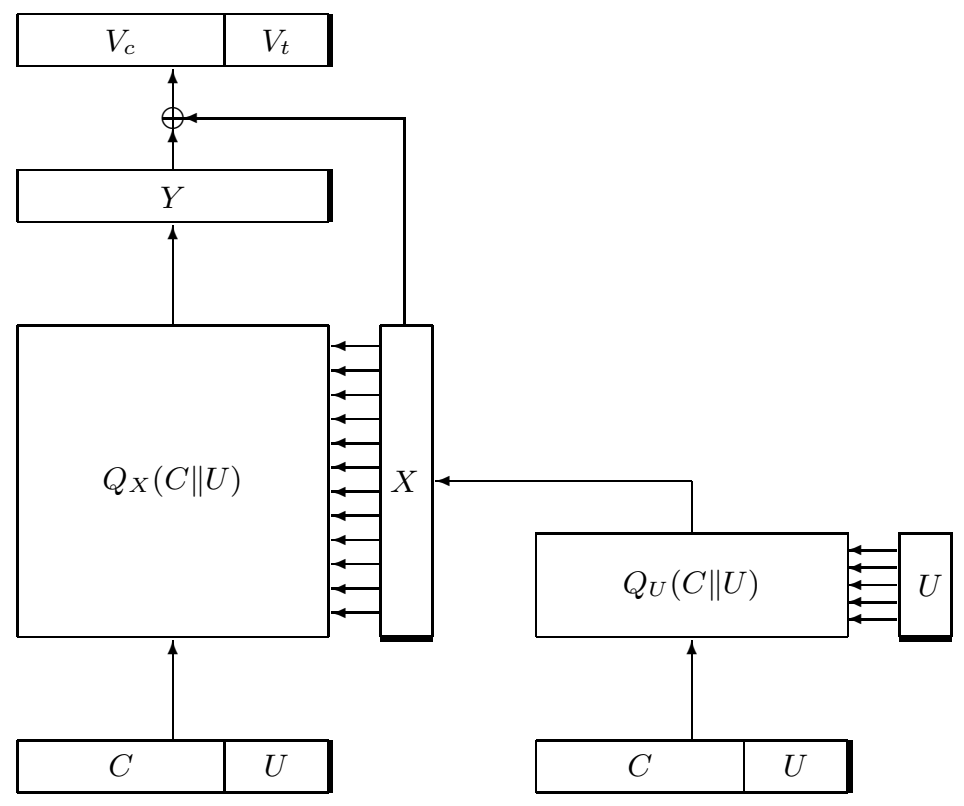

Fig. 1. The internal function of ARMADILL02. The thick line at the side of a register represents the least significant bit. 
We denote by cst a constant of size $k$ defined by alternating 0's and 1's, i.e. : $c s t=1010 \cdots 10$. Using these notations, we can specify $Q$ which is used twice in the internal ARMADILL02 function. Let $A$ be the $a$-bit parameter and $B$ be the $k$-bit input of $Q$, the parameterized permutation $Q_{A}$ can be divided into $a=|A|$ simple steps. The $i$-th step of $Q_{A}$ (reading $A$ from its least significant bit to its most significant one) is defined by:

- an elementary bitwise permutation: $B \leftarrow \sigma_{A[i]}(B)$, that is if the $i$-bit of $A$ is 0 we apply $\sigma_{0}$ to $B$, otherwise we apply $\sigma_{1}$.

- a constant addition (bitwise XOR) of cst: $B \leftarrow B \oplus$ cst.

The internal ARMADILL02 function first computes $X=Q_{U}(C \| U)$, then $Y=$ $Q_{X}(C \| U)$, and finally outputs $\left(V_{c}, V_{t}\right)=Y \oplus X$.

Using this internal primitive, ARMADILLO2 builds a FIL-MAC, a stream-cipher and a hash function:

- Stream-cipher: the secret key is inserted in the $C$ register and the output sequence is obtained by taking the $k$ bits of the output $\left(V_{c}, V_{t}\right)$ after one iteration. The keystream is composed of $k$-bit frames indexed by $U$ (which is a public value).

- Hash function: it uses a strengthened Merkle-Damgård construction, where $V_{c}$ represents the output of the compression function (i.e. the next chaining value or the hash digest), $U$ is the incoming message block and $C$ is the incoming chaining variable.

- FIL-MAC: the secret key is inserted in the $C$ register and the challenge, considered known by the attacker, is inserted in the $U$ register. The response to the challenge is the $m$-bit output $V_{t}$.

Five different sets of register sizes $(k, c, m)$ are provided, namely $(128,80,48)$, $(192,128,64),(240,160,80),(288,192,96)$ and $(384,256,128)$.

\section{First Tools}

We denote $\operatorname{HAM}(X)$ the Hamming weight of the word $X$. We recall from [1] that for two random $k$-bit words $A$ and $B$ of Hamming weight $a$ and $b$ respectively, the probability that $\operatorname{HAM}(A \wedge B)=i$ (where $\wedge$ stands for the bitwise AND function) is given by the formula

$$
P_{\text {and }}(k, a, b, i)=\frac{\left(\begin{array}{c}
a \\
i
\end{array}\right)\left(\begin{array}{c}
k-a \\
b-i
\end{array}\right)}{\left(\begin{array}{c}
k \\
b
\end{array}\right)}=\frac{\left(\begin{array}{l}
b \\
i
\end{array}\right)\left(\begin{array}{l}
k-b \\
a-i
\end{array}\right)}{\left(\begin{array}{l}
k \\
a
\end{array}\right)} .
$$

Moreover, we would like to deduce from it the probability that $\operatorname{HAM}(A \oplus B)=i$ (where $\oplus$ stands for the bitwise XOR function) for two randomly chosen $k$ bit words $A$ and $B$ of Hamming weight $a$ and $b$ respectively. We remark that $\operatorname{HAM}(A \oplus B)=a+b-2 \cdot \operatorname{HAM}(A \wedge B)$ and therefore the probability that $\operatorname{HAM}(A \oplus B)=$ $i$ is given by the formula. 


$$
P_{\text {xor }}(k, a, b, i)= \begin{cases}P_{\text {and }}\left(k, a, b, \frac{a+b-i}{2}\right) & \text { for }(a+b-i) \text { even } \\ 0 & \text { for }(a+b-i) \text { odd }\end{cases}
$$

Since they have not been specified in the original ARMADILL02 document, in the following we assume that $\sigma_{0}$ and $\sigma_{1}$ are randomly chosen bit permutations.

\section{Parity Preservation}

We call the parity bit of an $a$-bit word $A$ the bit value $\bigoplus_{i=0}^{a-1} A[i]$. Regardless of the parameter $A$ of the internal permutation $Q_{A}$, we have that the parity of the input is always maintained through the permutation. This can be easily verified by remarking that $Q_{A}$ is composed of several identical rounds, all satisfying this property. Indeed, one round is composed of a bit permutation (which fully maintains the Hamming weight) and an XOR of the internal state with the constant $c s t=1010 \ldots 10$. This constant being always the same during the whole ARMADILLO2 computation and its parity being even, the parity of the internal state remains the same after application of the XOR. Note that even if this constant was changed during the rounds, the attacker would only have to compute the parity of the XOR of all constants to be able to tell if the parity bit will be maintained or negated. This property is moreover maintained whatever number of rounds is applied in the permutations, thus the attack proposed in this section is independent of the number of rounds.

Distinguisher for the Stream Cipher Mode. We can exploit the previous property to build a cheap distinguisher on ARMADILLO2 when used as a streamcipher. In the attack model, the whole output of the function is assumed to be known as it is a frame of the keystream. This output is generated by a XOR of internal states $X$ and $Y$. Since permutations $Q_{U}$ and $Q_{X}$ will maintain the parity, their respective outputs $X$ and $Y$ will both have the same parity as $(C \| U)$. As a consequence, the output of the function $X \oplus Y$ always has an even parity. For a random sequence, this will only happen with probability $1 / 2$, as for ARMADILL02 this happens with probability 1. In other words, the entropy of the ARMADILLO2 function output is reduced by one bit.

\section{Controlled Diffusion: Practical Free-Start Collision Attack}

In this section, we show how an attacker can control the bit difference diffusion in ARMADILLO2 function by using the available inputs. This leads to a very cheap free-start collision attack against the compression function. 


\subsection{General Description}

Assume that we insert a single bit difference in $C$, that is $\operatorname{HAM}(\Delta C)=1$, and no difference in $U$ that is $\Delta U=0$. We can use $c$ distinct $\Delta C$, one for each active bit position. The attack is depicted in Figure 2 ,

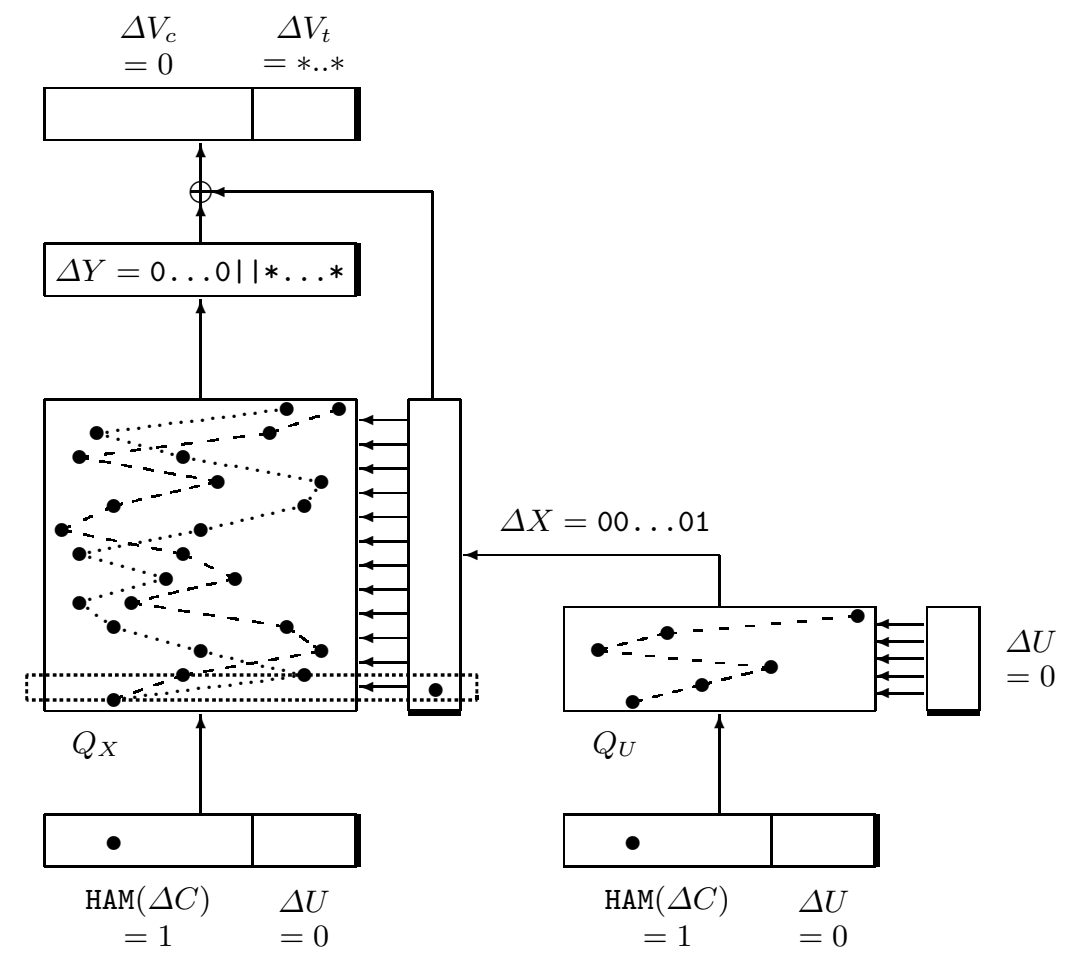

Fig. 2. A schematic view of the free-start collision attack on ARMADILLO2. The thick line at the side of a register represents the least significant bit and black circles stand for bit differences. The dashed box indicates the first round of $Q_{X}$, which contains a difference on its corresponding parameter input bit.

Difference Propagation in $Q_{U}$. Since we have no difference in $U$, the permutation $Q_{U}$ always remains the same. We only have to study the propagation of the bit difference in $C$ through $Q_{U}$. Note that one round of the internal permutation $Q_{U}$ provides no difference diffusion since it is only composed of a bit permutation and a constant addition. Therefore, the single bit difference in $C$ will be just transfered to some random bit position in $X$ at the end of $Q_{U}$ and we have $\operatorname{HAM}(\Delta X)=1$. We would like the single bit difference in $X$ to be positioned in bit 0 , i.e. $\Delta X=00 \ldots 01$ (this will later allow us to use the freedom degrees efficiently). For a randomly chosen value of $U$ and $C$, this happens with probability

$$
P_{X}=\frac{1}{k}
$$


Difference Propagation in $Q_{X}$. Since we have a single difference on the first bit of $X$ (corresponding to the first step of $Q_{X}$ ), the permutation $Q_{X}$ remains the same except for the first step where we switch from bit permutation $\sigma_{0}$ to $\sigma_{1}$ or from $\sigma_{1}$ to $\sigma_{0}$. We denote by $P_{\text {step }}($ in, out $)$ the probability that in active bits are mapped to out active bits through a step of data-dependent permutation with a difference (i.e. $\sigma_{0}$ and $\sigma_{1}$ are swapped). Assume for the moment that after this first step, only $b$ bits are active in the internal state. This happens with probability $P_{\text {step }}(1, b)$. Since the next rounds of the internal permutation $Q_{X}$ provide no difference diffusion, we end up in $Y$ with $b$ active bits randomly distributed. We need to ensure that all the $b$ active bits remaining in $Y$ will go to the $m$-bit $V_{t}$ part of the $k$-bit output, so that all differences will be truncated and we eventually obtain a collision on the output of the compression function. For $b \leq m$, this happens with probability

$$
P_{\text {out }}(b)=P_{\text {and }}(k, m, b, b)=\frac{\left(\begin{array}{l}
b \\
b
\end{array}\right)\left(\begin{array}{l}
k-b \\
m-b
\end{array}\right)}{\left(\begin{array}{l}
k \\
m
\end{array}\right)}=\prod_{i=0}^{i=b-1} \frac{m-i}{k-i} .
$$

During the feed-forward after $Q_{X}$ the single active bit of $X$ is already on the $V_{t}$ part of the output. Overall the probability of obtaining a compression function collision for randomly chosen $U$ and $C$ values is:

$$
P_{\text {collision }}=P_{X} \cdot \sum_{i=1}^{i=m} P_{\text {step }}(1, i) \cdot P_{\text {out }}(i) .
$$

the sum stopping at $m$ because when $i>m$, we trivially have $P_{\text {out }}(i)=0$. At this point our problem is that in order for the probability $P_{\text {out }}(i)$ to be high enough, we need the number $i$ of active bits to be small. On the other side, if $i$ is small, $P_{\text {step }}(1, i)$ will be very low (we do not explain how to compute $P_{\text {step }}(1, i)$ here as we will study a slightly more detailed problem in the next section). However, in this scenario we only considered an attacker that randomly chooses the value of $U$ and $C$ and the bit difference position in $C$, but we can do much better by using the available degrees of freedom efficiently.

\subsection{Using the Freedom Degrees}

First, note that the event related to the probability $P_{X}$ only depends on the position of the bit difference in $C$ and on the value of $U$. We can therefore attack $Q_{U}$ in a first phase (by fixing the position of the bit difference in $C$ and the value of $U$ ), and then independently attack $Q_{X}$ by choosing the value of $C$.

Handling $Q_{U}$. We will see later that we would like $C$ and $U$ values to have an extremely low or extremely high Hamming weight. Therefore, we fix $\Delta X=$ $00 \ldots 01$ and test with the two values $U=00 . .00$ and $U=11 . .11$ how the bit difference will propagate through $Q_{U}^{-1}$ (note that we are dealing with the inverse of $Q_{U}$, thus attacking backwards from $\left.\Delta X\right)$. For each try, we have a probability 
$P_{\text {and }}(k, c, 1,1)=c / k$ that the single bit difference is mapped to the $C$ part of the input. Since for all ARMADILL02 versions we have $2 c / k>1$, we expect at least one of the two $U$ candidates to satisfy $\Delta X=00 \ldots 01, \operatorname{HAM}(\Delta C)=1$ and $\operatorname{HAM}(\Delta U)=0$. Overall, this phase costs us only 2 operations. We assume without loss of generality that the selected candidate has value $U=00.00$.

Handling $\boldsymbol{Q}_{\boldsymbol{X}}$. At the present time, everything is fixed except the value of $C$ and we have $\Delta X=00 \ldots 01$ and $U=00 \ldots 00$. We now describe a simple criteria in order to choose the values of $C$ such that the first round probability $P_{\text {step }}(1, i)$ in $Q_{X}$ is high, even for small $i$. As an example, let's assume that $C=0$, that is $\operatorname{HAM}(C \| U)=0$. In that case, we trivially have that $P_{\text {step }}(1,1)=1$ (and $P_{\text {step }}(1, i)=0$ for all other $i$ ) since changing the bit positions of the word 00.00 (switching from $\sigma_{0}$ to $\sigma_{1}$ or from $\sigma_{1}$ to $\sigma_{0}$ ) will not have any effect at all and the single bit difference in $C$ will just be placed to some random bit position. Similarly, with a single one-bit in $C$, that is $\operatorname{HAM}(C \| U)=1$, we have that $P_{\text {step }}(1,1)=\frac{1}{128}+\frac{2 \cdot 127}{128^{2}}$ and $P_{\text {step }}(1,3)=\frac{127 \cdot 126}{128^{2}}$ (and $P_{\text {step }}(i)=0$ for all other $i$ ). More generally, we have to compute the probability $P_{\text {step }}(1, b, h w)$ which corresponds to the probability $P_{\text {step }}(1, b)$ knowing that the input word hamming weight is $h w$. This can be modeled as follows: choose two random $k$ bit words $x$ and $y$ both with Hamming weight $h w$ (they represent $\sigma_{0}(C|| U)$ and $\sigma_{1}(C \| U)$ ) and compute $z=x \oplus y \oplus 1$ (the 1 represents the single bit difference in $C$ ). Then $P_{\text {step }}(1, b, h w)$ is the probability that $\operatorname{HAM}(z)=b$ (note that $\operatorname{HAM}(z)$ is always odd thus we have $P_{\text {step }}(1,2 i, h w)=0$ for all $i$ ) and we have:

$$
P_{\text {step }}(1, b, h w)=\frac{h w}{c} \cdot P_{\mathbf{X o r}}(k, h w, h w-1, b)+\frac{c-h w}{c} \cdot P_{\mathbf{X o r}}(k, h w, h w+1, b) .
$$

The complexity for handling $Q_{X}$ is finally

$$
\text { Comp }=\frac{1}{\sum_{i=1}^{i=m} P_{\text {step }}(1, i, h w) \cdot P_{\text {out }}(i)}
$$

\subsection{Complexity Results}

The number $C$ of candidate values we can generate with Hamming weight $h w$ is $\left(\begin{array}{c}c \\ h w\end{array}\right)$ and in order to have a good chance to find a collision after $Q_{X}$ with this amount, we need to ensure that

$$
\left(\begin{array}{c}
c \\
h w
\end{array}\right) \geq 1 / \sum_{i=1}^{i=m} P_{\text {step }}(1, i, h w) \cdot P_{\text {out }}(i)
$$

One can check that in order to minimize the complexity Comp, the dominant factor of the sum is when $i$ is small. Then, for $i$ small, $P_{\text {step }}(1, i, h w)$ is higher when $h w$ is close to 0 or close to $k$, in other words the input should have very low or very high Hamming weight. Since we previously chose $U=00 \ldots 00$ our goal is to find for each ARMADILL02 versions the smallest $h w$ value $h w_{\text {min }}$ that 
ensures enough $C$ candidate values to handle the collision probability in $Q_{X}$ (but the same reasoning is possible with $U=11 \ldots 11$ and the biggest $h w$ value $\left.h w_{\max }\right)$. Overall, the full attack runs in $2+$ Comp operations (i.e. compression function calls) and negligible memory in order to find a free-start collision for the ARMADILLO2 compression function. We depict in Table 1 our results relative to all proposed versions of ARMADILL02. This attack has been implemented and verified in practice for $k=128$ and we give free-start collision examples in the Appendix.

Table 1. Summary of results for free-start collision attack on the different size variants of the ARMADILL02 compression function. The number of $C$ candidates must always be enough so as to handle the collision probability in $Q_{X}$.

\begin{tabular}{|c|c|c||c||c|c|c||c|}
\hline \multicolumn{3}{|c||}{ scheme parameters } & \multicolumn{4}{c|}{ attack parameters } \\
\hline$k$ & $c$ & $m$ & $\begin{array}{c}\text { generic } \\
\text { complexity }\end{array}$ & $h w_{\min }$ & $\begin{array}{c}\text { nber of } C \\
\text { candidates }\end{array}$ & $\begin{array}{c}\text { collision } \\
\text { prob. in } Q_{X}\end{array}$ & $\begin{array}{c}\text { attack } \\
\text { complexity }\end{array}$ \\
\hline \hline 128 & 80 & 48 & $2^{40}$ & 1 & $2^{6.3}$ & $2^{-4.1}$ & $2^{7.5}$ \\
\hline 192 & 128 & 64 & $2^{64}$ & 1 & $2^{7}$ & $2^{-4.6}$ & $2^{7.8}$ \\
\hline 240 & 160 & 80 & $2^{80}$ & 1 & $2^{7.3}$ & $2^{-4.7}$ & $2^{8.1}$ \\
\hline 288 & 192 & 96 & $2^{96}$ & 1 & $2^{7.6}$ & $2^{-4.7}$ & $2^{8.3}$ \\
\hline 384 & 256 & 128 & $2^{128}$ & 1 & $2^{8}$ & $2^{-4.8}$ & $2^{8.7}$ \\
\hline
\end{tabular}

\section{Local Linearization: Practical Semi-free-Start Collision Attack}

In this section, we show how one can obtain a semi-free-start collision attack (no difference on the input chaining variable) with a very low computational complexity for the ARMADILLO2 compression function.

\subsection{General Description}

The previous method only allows to add differences on the capacity part of the input, thus leading to free-start collision attacks. One can directly extend this technique to allow only differences in the message part of the input, but this only leads to semi-free-start collisions for randomly chosen bit permutations $\sigma_{0}$ and $\sigma_{1}$ with a not-so-high probability of success.

We would like to derive a semi-free-start collision attack that will output a result with very high probability. In order to achieve this goal we propose a new technique for data-dependent bit transposition ciphers, so-called local linearization: by guessing some part of the input we are able to render a few rounds of the internal permutation linear. Indeed, by knowing the $g$ first bits of 
$U$ we completely determine the permutations applied during the first $g$ rounds of $Q_{U}$. Therefore, for those $g$ rounds the primitive $Q_{U}$ only consists of known bit permutations and known constant additions. With this method we neutralize for the first $g$ rounds the only non-linearity source: the fact that we don't know which bit permutation $\sigma_{0}$ or $\sigma_{1}$ is applied each round.

On a high-level view, our semi-free-start collision attack will force a collision on the $X$ value at the output of $Q_{U}$ thanks to the local linearization technique. This collision on $X$ will ensure that the $Q_{X}$ permutation will be the same for both inputs. Therefore, the difference Hamming weight on the input of $Q_{X}$ will remain the same in the output. We then hope that those bit differences will be mapped in the truncated part of the output in order to eventually obtain the semi-free-start collision (no difference is feed-forwarded from $X$ since we forced a collision on it). The attack is depicted in Figure 3 .

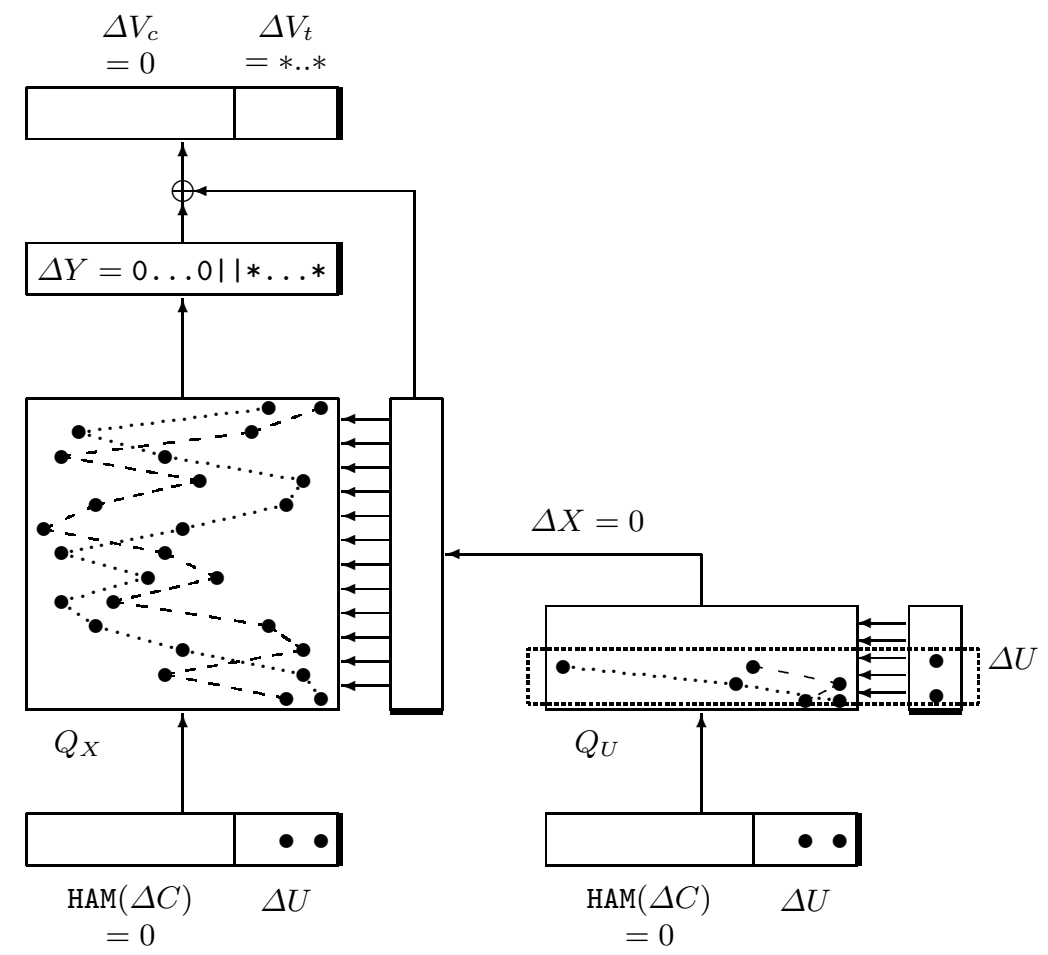

Fig. 3. A schematic view of the semi-free-start collision attack on ARMADILLO2. The thick line at the side of a register represents the least significant bit and black circles stand for bit differences. The dashed box indicates the linearized part.

During a first phase, the input will be divided into two parts: the fixed and the unfixed part. The fixed part $z \in\{0,1\}^{g}$ is composed of the $g$ first bits of $U$ and we choose random values for those $g$ bits (so as to know the $g$ first choices of $\sigma_{0}$ or $\left.\sigma_{1}\right)$. The unfixed part $w \in\{0,1\}^{k-g}$ is composed of the rest of the input bits and 
we will be set to a value later. We force the input difference to be contained in the fixed part and we denote it $\Delta z \in\{0,1\}^{g}$ (since we are looking for semi-freestart collisions we obviously have $g \leq m$, otherwise we would have a difference in the input chaining variable $C$ ). Let $I_{1}=\left(C_{1} \| U_{1}\right)$ (resp. $\left.I_{2}=\left(C_{2} \| U_{2}\right)\right)$ be the $k$-bit value of the first input (resp. second output), we have:

$$
I_{1}=(x \| z) \text { and } I_{2}=(x \| z \oplus \Delta z) \text {. }
$$

and our goal is to have the collision $X=Q_{U_{1}}\left(I_{1}\right)=Q_{U_{2}}\left(I_{2}\right)$.

Assume for the moment that this collision on $X$ happens. Then the same permutation $Q_{X}$ will be used for both inputs $I_{1}$ and $I_{2}$ on the right side of Figure 1 As a consequence, no additional bit difference will be introduced during the computation of $Q_{X}$, but the bit difference positions will be randomly moved. In order to obtain a semi-free-start collision on the output of the function, we need the $b=\operatorname{HAM}(\Delta z)$ active bits of the input to be mapped in the truncated part of the output through $Q_{X}$. As already explained in Section 5, this happens with probability

$$
P_{\text {out }}(b)=P_{\text {and }}(k, m, b, b)=\prod_{i=0}^{i=b-1} \frac{m-i}{k-i} .
$$

\subsection{Colliding on $X$}

We need now to evaluate the probability of getting a collision on $X$. Note that for any round, if there is no difference on the bit choosing the permutation to apply $\sigma_{0}$ or $\sigma_{1}$, the bit differences at the input of this round will only have their position changed and cannot be erased. Therefore, if we want to obtain a collision on $X$, we need to obtain it at latest just after the last round of $Q_{U}$ for which a difference is inserted on the side (in $U$ ). We consider from now on that the input difference $\Delta z$ contains at least one active bit on its MSB, thus this last round is the $g$-th one.

We know the value of the $g$ first bit of $U$, therefore we know exactly the permutation applied to $I_{1}$ and $I_{2}$ for the $g$ first rounds of $Q_{U}$. For a collision after $g$ rounds of $Q_{U}$, we want that

$$
\begin{aligned}
& \sigma_{U_{1}[g-1]}\left(\cdots\left(\sigma_{U_{1}[1]}\left(\sigma_{U_{1}[0]}\left(I_{1}\right) \oplus c s t\right) \oplus c s t\right) \cdots\right) \\
= & \sigma_{U_{2}[g-1]}\left(\cdots\left(\sigma_{U_{2}[1]}\left(\sigma_{U_{2}[0]}\left(I_{2}\right) \oplus c s t\right) \oplus c s t\right) \cdots\right)
\end{aligned}
$$

and since all operations are linear, this can be rewritten as

$$
\rho\left(I_{1}\right) \oplus A=\rho^{\prime}\left(I_{2}\right) \oplus B=\rho^{\prime}\left(I_{1} \oplus \Delta z\right) \oplus B=\rho^{\prime}\left(I_{1}\right) \oplus \rho^{\prime}(\Delta z) \oplus B
$$

where

$$
\begin{array}{ll}
\rho=\sigma_{U_{1}[g-1]} \circ \cdots \sigma_{U_{1}[1]} \circ \sigma_{U_{1}[0]} & A=\sigma_{U_{1}[g-1]}\left(\cdots\left(\sigma_{U_{1}[1]}(c s t) \oplus c s t\right) \cdots\right) \\
\rho^{\prime}=\sigma_{U_{2}[g-1]} \circ \cdots \sigma_{U_{2}[1]} \circ \sigma_{U_{2}[0]} & B=\sigma_{U_{2}[g-1]}\left(\cdots\left(\sigma_{U_{2}[1]}(c s t) \oplus c s t\right) \cdots\right) .
\end{array}
$$


Finally, we end up with the equation

$$
\rho\left(I_{1}\right) \oplus \rho^{\prime}\left(I_{1}\right)=A \oplus B \oplus \rho^{\prime}(\Delta z)
$$

Since we know the value of the $g$ first bit of $U$, we can compute the value of $A$ and $B$. Moreover, assuming that we already chose a $\Delta z$, then the collision condition (1) can be rephrased as

$$
I_{1} \oplus \tau\left(I_{1}\right)=C
$$

where $C=\rho^{-1}\left(A \oplus B \oplus \rho^{\prime}(\Delta z)\right)$ and $\tau=\rho^{-1} \circ \rho^{\prime}$.

In order to study this system $\mathcal{S}$ of $k$ bit equations, we model $\tau$ as a random bit permutation and $C$ as a random $k$-bit word. Note that since this equation system is linear finding the potential solutions requires only a few operations, but we would like to know how many such systems we need to generate before finding a solution, i.e. a collision on $X$. Thus, our goal is now to deduce the probability that this system has at least one solution and what is the average number of expected solutions.

The structure of this equation system is very particular and the number of independent groups of bit equations is exactly the number of cycles of the bit permutation $\tau$. More precisely, let $\operatorname{CYCLE}(\tau)$ represent the number of cycles of the permutation $\tau$ and let $S_{i}$ denote the set of bits belonging to the $i$-th cycle of $\tau$.

Theorem 1. The equation system $\mathcal{S}: I_{1} \oplus \tau\left(I_{1}\right)=C$ admits a solution if and only if for every cycle set $S_{i}$ of $\tau$ the parity of the sum of the corresponding $C$ bit is null, that is

$$
\bigoplus_{p \in S_{i}} C[p]=0
$$

If this system is solvable, then the number of solutions that can be generated is exactly equal to $2^{\mathrm{CYCLE}(\tau)}$.

The Idea of the Theorem is that when we want to find a solution for the system, we can start by fixing one bit $a_{0}$ to a random value. This bit is involved into two binary equations from $\mathcal{S}$. All equations having only two terms, one of the two equations directly links bit $a_{0}$ with say bit $a_{1}$, and we can deduce the value of $a_{1}$. The bit $a_{1}$ is in turn linked with bit $a_{2}$ through his second equation and we directly deduce the value of $a_{2}$. This chain of dependency will eventually cycle (the new bit deduced will be $a_{0}$ again) and will be validated if and only if the sum of the $C$ bits of the equations visited is null (otherwise we encounter a inconsistency). This check is then performed for all cycles.

Proof. Since $\tau$ is a bit permutation, the equation system $\mathcal{S}$ can be represented as a collection of cycles, each cycle depicting the direct cyclical dependencies between some set of bits: if bit $x$ and bit $y$ are linked by one of the $k$ equations, 
then they belong to the same cycle. The vertex weight between two members $x$ and $y$ of the cycle is the value $C[x]$.

If we fix the bit value of a member of a cycle $S_{i}$, then this determines entirely all the other bits of that cycle (according to the vertices values). Then, if the XOR of all the vertex weights is different from zero, we have a direct contradiction. A solution can only exist if all cycles present no internal contradiction.

Each cycle can have either zero or two solutions (the two solutions being their mutual complement). If every cycle has no contradiction, then there exists exactly $2^{\mathrm{CYCLE}(\tau)}$ distinct combinations of cycle solutions, each one leading to a distinct solution for the whole equation system $\mathcal{S}$.

From Theorem 1, we directly deduce that the probability that the system admits a solution is equal to $2^{-\operatorname{CYCLE}(\tau)}$. The expected number of cycles for a randomly chosen permutation on $k$ elements is $\log (k)$. Therefore, we have to try at least $2^{\log (k)}$ different equation systems before finding one admitting a solution. When one system admits a solution, we directly get $2^{\log (k)}$ solutions for free. Overall, the cost for finding one solution of the system is 1 on average (the average cost is the meaningful one here since we will have to find several inputs colliding on $X$ during the whole attack).

\subsection{Complexity Results}

We now look for a solution such that the original guess of the $g$ first bits of the input was right (with probability $2^{-g}$ ) and such that the $b$ bit differences in $Q_{X}$ are mapped to the truncated part of the output (with probability $P_{\text {out }}(b)$ ). Overall, the total complexity of the semi-free-start collision attack is $2^{g} \cdot P_{\text {out }}^{-1}(b)$ with $b \leq g$. Minimizing $g$ and $b$ will minimize the overall complexity, but we need to ensure that we can go through enough equation systems in order to have a good chance to find a collision eventually. More precisely, we need

$$
1 / 2 \cdot 2^{g} \cdot\left(\begin{array}{l}
g \\
b
\end{array}\right) \geq 2^{g} \cdot P_{\text {out }}^{-1}(b)
$$

which can be rewritten as

$$
\left(\begin{array}{l}
g \\
b
\end{array}\right) \geq 2 \cdot P_{\text {out }}^{-1}(b) .
$$

We depict in Table 2 our results relative to all proposed versions of ARMADILL02. This attack has been implemented and verified in practice for $k=128$ and we give semi-free-start collision examples in the Appendix.

\section{Related-Key Recovery in Stream Cipher Mode}

In this section we will present a related key attack that will allow us to recover all key bits in practical time when using ARMADILLO2 in the stream cipher mode. We will first present the main idea of this attack, and afterwards, we will give a more detailed analysis of the probabilities and complexities. 
Table 2. Summary of results for semi-free-start collision attack on the different size variants of the ARMADILLO2 compression function

\begin{tabular}{|c|c|c||c||c|c|c||c|}
\hline \multicolumn{4}{|c||}{ scheme parameters } & \multicolumn{3}{|c|}{ attack parameters } \\
\hline$k$ & $c$ & $m$ & $\begin{array}{c}\text { generic } \\
\text { complexity }\end{array}$ & $g$ & $b$ & $P_{\text {out }}(b)$ & $\begin{array}{c}\text { time } \\
\text { complexity }\end{array}$ \\
\hline \hline 128 & 80 & 48 & $2^{40}$ & 6 & 2 & $2^{-2.9}$ & $2^{8.9}$ \\
\hline 192 & 128 & 64 & $2^{64}$ & 7 & 2 & $2^{-3.2}$ & $2^{10.2}$ \\
\hline 240 & 160 & 80 & $2^{80}$ & 7 & 2 & $2^{-3.2}$ & $2^{10.2}$ \\
\hline 288 & 192 & 96 & $2^{96}$ & 7 & 2 & $2^{-3.2}$ & $2^{10.2}$ \\
\hline 384 & 256 & 128 & $2^{128}$ & 7 & 2 & $2^{-3.2}$ & $2^{10.2}$ \\
\hline
\end{tabular}

\subsection{Using Related-Keys for Recovering the Key}

First of all, we consider a pair of related keys $\left(K_{1}, K_{2}\right)$ that have one only bit of difference, that is $\operatorname{HAM}\left(K_{1} \oplus K_{2}\right)=\operatorname{HAM}\left(\Delta_{K}\right)=1$. Our analysis will work for any bit difference position $d$ amongst all the bits of the key. Note that we expect a pair of keys valid for performing the related-key attack to appear after using about $\left(2^{k} / k\right)^{1 / 2}$ keys.

Let us consider a value of $U$ for generating $k$ bits of key-stream with each of both keys $K_{1}$ and $K_{2}$. We use the index $i$ for the intermediate states generated from the key $K_{i}$. We first make the following observations, important in order to understand the whole attack procedure:

- Since no difference is inserted in the $U$ part (it is a public value) and since $\operatorname{HAM}\left(\Delta_{K}\right)=1$, we have $\operatorname{HAM}\left(X_{1} \oplus X_{2}\right)=1$. Let $e$ be the bit position of this difference in $X$.

- The first $(e-1)$ intermediate states of $Q_{X}$ will also have a difference of Hamming weight 1.

We assume that the attacker can choose the values of $U$. In this case, we can make the bit difference in the key to go from position $d$ to any wanted position $e$ in $X$ through $Q_{U}$. We expect $2^{m} / k$ distinct values of $U$ that make the bit difference go from position $d$ to $e$ for $e \in[0, k-1]$. We denote by $U_{e}$ each one of these $k$ subgroups of $U$ values.

The output of the function $\left(V_{c}, V_{t}\right)=X \oplus Y$ is known to the attacker, but concerning $X$ he only knows the $m$ bits of the $U$ part (since $U$ is known, he can deduce directly where the bits coming from $U$ and $C$ will be eventually located in $X)$. Thus, he can recover $m$ bits from the outputs of $Q_{X}, Y_{1}$ and $Y_{2}$. If he could compute backward from $Y_{1}$ and $Y_{2}$ until the beginning of the $e$-th step of $Q_{X}$, the colliding positions of the bits known from $Y_{1}$ and from $Y_{2}$ will have the same values with maybe the exception of one, which would be the original single bit difference before the step $e$. 
Our attack basically consists in choosing several values for $U$ from $U_{e}$, for decreasing $e$ values (starting from $e=k-1$ ), that will gradually increase the number of key bits appearing in $X$ after position $e$. Each time we will guess the value of the new key bits appearing and discard the guesses that will not lead to collisions on the bit values in the colliding positions just before step $e$ when computing backward from $Y_{1}$ and $Y_{2}$ in $Q_{X}$. The complexity of this attack depends on the bit permutations $\sigma_{0}$ and $\sigma_{1}$, but in the next subsection we give a complexity analysis assuming that these permutations are randomly chosen.

\subsection{Generic Complexity Estimation}

We start at $e=k-1$. First, we choose the value of $i$ (denoted $i_{\max }$ ), that maximizes the probability $P_{\text {and }}(k, m, m, i)$ that we denote $p_{\max }$. For instance, if we consider the smallest version of ARMADILLO2, where $k=128, c=80$ and $m=48$, then we have $i_{\max }=18$ and the probability of obtaining 18 positions of known bits that collide is equal to $p_{\max }=2^{-2.72}$.

Amongst the values from $U_{k-1}$, we choose $p_{\max }{ }^{-1}$ random ones. Each of them is introduced in the ARMADILLO2 function parametrized with the keys $K_{1}$ and $K_{2}$. For each of the $p_{\max }{ }^{-1}$ pairs of values, we guess the bit at position $k-1$ of $X_{1}$ and of $X_{2}$ (for example 1 and 0 respectively since there is a difference on this bit position) and we end up with $2 \cdot p_{\max }{ }^{-1}$ pairs. Then, we can undo the last round of $Q_{X}$ for the known bits from $Y_{1}$ and $Y_{2}$. We consider that a guess passes the test if it verifies the conditions on the number of colliding values on the colliding bit positions. For one of these $2 \cdot p_{\max }{ }^{-1}$ pairs (in our example $\left.\left(Q_{1}^{-1}\left(Y_{1}\right), Q_{0}^{-1}\left(Y_{2}\right)\right)\right)$, the number of colliding bit positions will be $i_{\max }$. When this is the case, if the guess on the bit of $X_{1}$ and $X_{2}$ was incorrect, we have a probability of $2^{-i_{\max }+1}$ to pass the test, while we will pass it with probability one if the guess was correct. Finally, we have determined one bit of each key $K_{1}$ and $K_{2}$ with a complexity of $2 \cdot p_{\max }{ }^{-1}$, which in our example would be $2^{3.72}$.

We can continue the process by considering $e=k-2$ and $p_{\max }{ }^{-1}$ values from $U_{k-2}$ that have a key bit at position $k-1$. Following the same method as before, we will recover one key bit, i.e. the one at position $k-1$ in $X$ when we have 18 colliding bits before the step $k-1$ of $Q_{X}$. Let us remark here that in practice we do not have to wait for having a collision on 18 bits, but most of the time collisions on a different number of bits will also be enough for determining if a guess passes the test or not. We can repeat this step in order to obtain the biggest possible number of key bits and determining each bit will add at most a complexity of $p_{\max }{ }^{-1}$.

The next steps depend on the number of bits that we have already determined. All in all, we conjecture that when both bit permutations behave like random ones, the complexity will not exceed $2 \cdot c \cdot p_{\max }{ }^{-1}$.

\section{Conclusion}

We have presented some new and practical analysis of ARMADILLO2. Notably a free-start and semi-free-start collision attacks for the full ARMADILL02 hash 
functions. Extending this work to real collisions (i.e. with a predefined IV) might be possible but it is not very appealing because it is likely that several message blocks are required (all versions have $c>m$ ) and therefore the task of the cryptanalyst would be quite complex to handle. ARMADILLO2 should not be used in any security application since our attacks have a very low complexity. This work and the local-linearization method is a first step in order to evaluate the security of data-dependent bit transpositions cryptographic designs.

Acknowledgements. The authors would like to thank the anonymous referees and the ARMADILLO2 team for their helpful comments.

\section{References}

1. Abdelraheem, M.A., Blondeau, C., Naya-Plasencia, M., Videau, M., Zenner, E.: Cryptanalysis of ARMADILLO2. In: Lee, D.H., Wang, X. (eds.) ASIACRYPT 2011. LNCS, vol. 7073, pp. 308-326. Springer, Heidelberg (2011)

2. Badel, S., Dağtekin, N., Nakahara Jr., J., Ouafi, K., Reffé, N., Sepehrdad, P., Sušil, P., Vaudenay, S.: ARMADILLO: A Multi-purpose Cryptographic Primitive Dedicated to Hardware. In: Mangard, S., Standaert, F.-X. (eds.) CHES 2010. LNCS, vol. 6225, pp. 398-412. Springer, Heidelberg (2010)

3. Brassard, G. (ed.): CRYPTO 1989. LNCS, vol. 435. Springer, Heidelberg (1990)

4. Damgård, I.: A Design Principle for Hash Functions. In: Brassard [3], pp. 416-427

5. Lai, X., Massey, J.L.: A Proposal for a New Block Encryption Standard. In: Damgård, I.B. (ed.) EUROCRYPT 1990. LNCS, vol. 473, pp. 389-404. Springer, Heidelberg (1991)

6. Merkle, R.C.: One Way Hash Functions and DES. In: Brassard [3], pp. 428-446

7. Rivest, R.L.: The RC5 Encryption Algorithm, pp. 86-96. Springer (1995)

8. Rivest, R.L., Robshaw, M.J.B., Yin, Y.L.: RC6 as the AES (2000)

9. Sepehrdad, P., Sušil, P., Vaudenay, S.: Fast Key Recovery Attack on ARMADILLO1 and Variants. In: Prouff, E. (ed.) CARDIS 2011. LNCS, vol. 7079, pp. 133-150. Springer, Heidelberg (2011)

\section{A Implementation of the Collision Attacks for $k=128$}

We implemented all attacks for $k=128$ and they require less than a second and negligible memory on an average computer (Intel Core2 Duo CPU @ $2.13 \mathrm{GHz}$ ) in order to find a collision. Since no specific $\sigma_{0}$ and $\sigma_{1}$ bit transpositions are defined for ARMADILL02, we run the attack for many randomly chosen instances so as to ensure the soundness of our reasoning. We give here examples of (semi)free-start collisions for ARMADILLO2 with a $\sigma_{0}$ and $\sigma_{1}$ bit transpositions instance that fulfill the criteria required in [2] for $k=128$. Namely, we denote $\lambda$ the second largest eigenvalue of the matrix $M=\frac{1}{4}\left(P_{\sigma_{0}}+P_{\sigma_{0}}^{128}+P_{\sigma_{1}}+P_{\sigma_{1}}^{128}\right)$, then for the $\sigma_{0}$ and $\sigma_{1}$ instance found we have $\lambda=0.87$. This means that there exists a distinguisher with advantage $\lambda^{256}=2^{-51.4}$, while our attacks have much better advantage. 
Free-Start Collision for ARMADILL02 with $k=128, c=80, m=48$ :

ARMADILL02(ffffffffffffffffbfff,ffffffffffff) $=$ ARMADILLO2(fffffdffffffffffbfff, ffffffffffff) $=$ dfb0d8f2b763ce97f 785

Semi-Free-Start Collision for ARMADILL02 with $k=128, c=80, m=48$ :

$$
\begin{array}{r}
\text { ARMADILL02(6bc8c848de5ff533cd6f, } 0850 \mathrm{~b} 04 \mathrm{~b} 82 \mathrm{e} 2)= \\
\text { ARMADILL02(6bc8c848de5ff533cd6f, 0850b04b82f0) }= \\
26827 \mathrm{e} 3 \mathrm{~d} 614 \mathrm{~d} 2 \mathrm{fc75d64}
\end{array}
$$

\section{Bit Transpositions $\sigma_{0}$ and $\sigma_{1}$ Used:}

$\sigma_{0}=62,98,14,114,36,77,55,3,28,88,29,122,57,90,66,52,44,22,95,118,69,86$, $35,56,58,82,18,97,78,21,85,101,19,65,10,6,116,121,70,99,61,102,4,91$, $39,119,79,16,84,50,113,45,93,104,73,112,8,5,51,9,105,46,64,94,41,54$, $127,67,106,23,63,49,123,15,60,81,96,72,110,37,30,89,7,92,2,68,40,32$, $53,11,71,26,103,59,109,111,38,74,20,48,24,43,126,117,13,124,31,33$, $100,125,87,27,83,128,12,42,80,107,108,17,25,120,76,75,115,47,1,34$

$\sigma_{1}=10,60,111,78,38,57,110,75,104,56,88,79,23,99,16,22,128,94,120,24,64$, $3,6,55,42,51,43,82,114,89,26,35,61,73,77,36,28,21,105,15,67,70,113$, $65,39,80,122,31,101,100,107,124,18,46,85,19,49,14,12,71,86,68,102,91$, $58,95,1,53,83,125,66,98,81,44,48,59,27,9,119,40,45,74,92,112,93$, $69,5,108,106,115,90,13,84,126,7,109,54,127,33,121,62,87,30,29,63,2$, $97,116,4,47,11,8,34,96,118,72,52,103,37,25,123,50,76,17,20,41,117,32$ 